\title{
The proposed new SI: consequences for mass metrology
}

\author{
Roman Schwartz, Michael Borys \\ Physikalisch-Technische Bundesanstalt, Bundesallee 100, 38116 Braunschweig, Germany
}

ABSTRACT

At its $24^{\text {th }}$ meeting in October 2011 the General Conference on Weights and Measures (CGPM) adopted a Resolution on the possible future revision of the International System of Units (SI). This paper provides an overview of the proposed changes to the SI, the focus being on the proposed redefinition of the kilogram and possible consequences for mass metrology.

Section: RESEARCH PAPER

Keywords: New SI; redefinition; kilogram; mass.

Citation: Roman Schwartz, Michael Borys, The proposed new SI: consequences for mass metrology, Acta IMEKO, vol. 3, no. 2, article 3, June 2014, identifier: IMEKO-ACTA-03 (2014)-02-03

Editor: Paolo Carbone, University of Perugia

Received February $14^{\text {th }}, 2014$; In final form May 25 ${ }^{\text {th }}, 2014$; Published June 2014

Copyright: (C) 2014 IMEKO. This is an open-access article distributed under the terms of the Creative Commons Attribution 3.0 License, which permits unrestricted use, distribution, and reproduction in any medium, provided the original author and source are credited

Funding: (none reported)

Corresponding author: Roman Schwartz, e-mail: Roman.Schwartz@ptb.de

\section{INTRODUCTION}

Of the seven base units of the SI, only the kilogram is still defined in terms of a material artefact, namely the international prototype of the kilogram (IPK) kept at the BIPM [1]. Since the third verification of national prototypes of the kilogram (NPK) against the IPK in the period 1989 to 1991 the stability of the IPK has been put into question, because the results of comparisons between the NPKs and the IPK show some divergence with time, the relative mass changes being in the order of $50 \mu \mathrm{g}$ during a period of about 100 years. Although the mass of the IPK is certainly not stable, neither could its drift yet be determined absolutely with sufficiently small uncertainty nor could it be clarified whether the observed mass changes are primarily due to a drift of the NPKs, or of the IPK, or of both.

Unknown changes in the mass unit also influence the electrical units, because the definition of the ampere is related to the kilogram, see Figure 1. Similarly, the definitions of the mole and candela also depend on the kilogram.

In 1999 the CGPM therefore recommended that efforts be continued to refine experiments linking the unit of mass to fundamental constants with sufficiently high accuracy (some parts in $\left.10^{8}\right)$, which preserves continuity in practical mass determination after a new definition of the kilogram is introduced.

\section{PROPOSED CHANGES TO THE SI}

At its 24th meeting in 2011, the CGPM adopted its Resolution 1 "On the possible future revision of the
International System of Units, the SI" [2] in which the CGPM takes note of the intention of the International Committee for Weights and Measures (CIPM) to redefine not only the kilogram, but all seven SI base units, in terms of invariants of nature and to express all definitions uniformly. With this, the CGPM and the CIPM clearly intend to revise the SI with a view that it continues to meet the needs of science, technology, and commerce in the $21^{\text {st }}$ century.

The proposed changes to the SI can be summarized as follows [3].

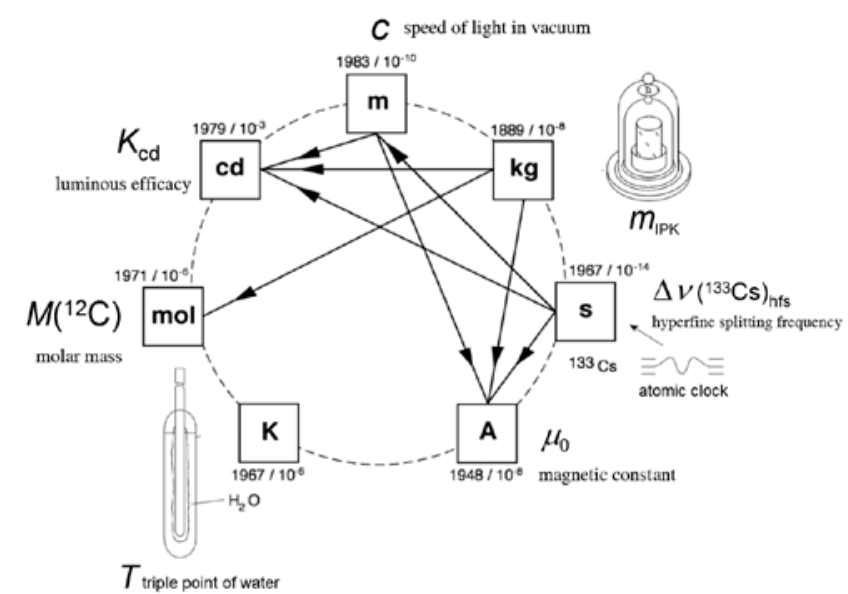

Figure 1. The seven base units and their relationship in the current SI. 


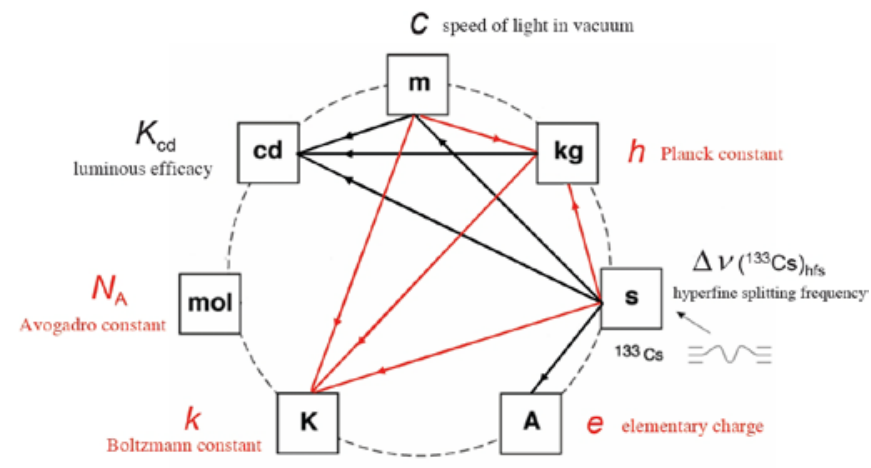

Figure 2. Definition of and relationship between the seven base units in the proposed new SI. In the new SI all base units will be defined in terms of fundamental or atomic constants. The changes to the current $\mathrm{SI}$, and the new relationships, are marked in red. The black arrows denote relationships that remain unchanged in the new SI.

- Keep the existing seven SI base units, but define them all in terms of seven well-recognized fundamental or atomic constants, such as the Planck constant $h$, see Figure 2.

- Fix the values of all these constants to an exact number (with zero uncertainty), as is already the case for the speed of light in vacuum, c = 299792458 metre per second.

- Use "explicit-constant" formulations to express the definitions of all seven SI base units in a uniform (but indirect) manner.

- Draw up specific "mise en pratique" (i.e. sets of instructions) for each base unit to explain how the units can be practically realized based on recommended top-level methods.

The kilogram would be defined in terms of the Planck constant $h$, the ampere in terms of the elementary charge $e$, the kelvin in terms of Boltzmann's constant $k$, and the mole in terms of the Avogadro constant $N_{\mathrm{A}}$.

The second would still be defined in terms of the hyperfine splitting frequency of the ground state of the caesium 133 atom, $\Delta v\left({ }^{133} \mathrm{Cs}\right)_{\mathrm{hfs}}$, but the formulation would be changed into an "explicit-constant" one, where the unit (here the second) would be defined indirectly by specifying explicitly an exact value for $\Delta v\left({ }^{133} \mathrm{Cs}\right)$, namely $9192631770 \mathrm{~Hz}$. The same would hold for the metre (defined in terms of the speed of light in vacuum $c$ ), and the candela (defined in terms of the luminous efficacy $K_{\mathrm{cd}}$ of monochromatic radiation of frequency $540 \mathrm{THz}$ ).

The CGPM Resolution 1 (2011) highlights the following advantages of such a "new SI":

- The uncertainties of all SI electrical units together with the SI values of the Josephson and von Klitzing constants $K_{\mathrm{J}}$ and $R_{K}$ would be significantly reduced.

- The kelvin would no longer be defined in terms of an intrinsic property of water that, while being an invariant of nature, in practice depends on the purity and isotopic composition of the water used.

- The mole would no longer depend on the definition of the kilogram; this would emphasize the distinction between the quantities "amount of substance" and "mass".

- The uncertainties of the values of many other important fundamental constants and energy conversion factors would be eliminated or greatly reduced.

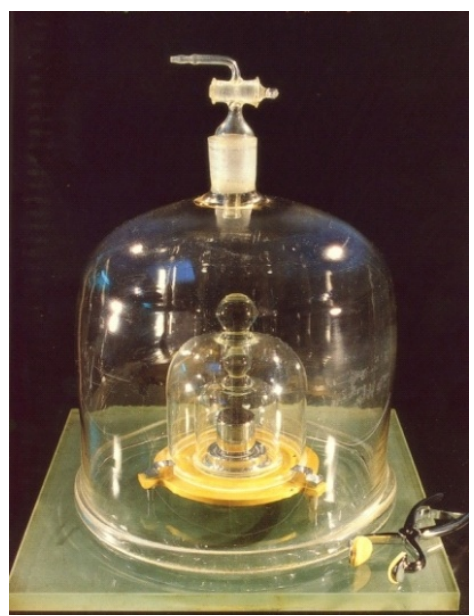

Figure 3. The IPK is kept at the BIPM in Sèvres. It still defines the unit of mass, the kilogram. Its mass, $m_{\mathrm{IPK}}$, is defined to be exactly $1 \mathrm{~kg}$ with zero uncertainty. In the proposed new $\mathrm{SI}$ it will have non-zero uncertainty (source: BIPM, Sèvres, France).

\section{REDEFINITION OF THE KILOGRAM}

In the present SI, one kilogram is defined as exactly the mass of the IPK, see Figure 3.

According to draft chapter 2 of the $9^{\text {th }}$ SI brochure [4], the new explicit-constant definition of the kilogram would read:

"The kilogram, kg, is the unit of mass; its magnitude is set by fixing the numerical value of the Planck constant to be equal to exactly $6.62606 \mathrm{X} \times 10^{-34}$ when it is expressed in the unit $\mathrm{s}^{-1} \mathrm{~m}^{2}$ $\mathrm{kg}$, which is equal to $\mathrm{J} \mathrm{s} . "$

The exact value for $\mathrm{X}$, which will be fixed by the latest CODATA adjustment at the time of the redefinition [5], requires further experimental effort to reach a sufficiently small relative measurement uncertainty in the order of $10^{-8}$.

The Consultative Committee for Mass and Related Quantities (CCM) is currently drafting a mise en pratique for the redefined kilogram. This will explain how the kilogram can be realized in the future by different primary methods (e.g. the "Avogadro method", also known as "X-ray crystal density (XRCD) method", or the "Watt balance method") using primary mass standards, and how the NPK or other secondary mass standards of National Metrology Institutes (NMI) can be linked to the primary mass standards and the Planck constant $h$, see Figures 4 and 5.

Compared with the redefinition of other base units the redefinition of the kilogram is the most critical one, for several reasons:

- Accurate weighings and mass determinations are of extraordinary importance in science, trade and industry,

- There are partly very high demands on the accuracy of mass determinations. Class $\mathrm{E}_{1}$ [7] accredited mass laboratories, for instance, keep reference standards with relative uncertainties between $2.5 \times 10^{-8}$ and $5 \times 10^{-8}$,

- If the value for the Planck constant $b$ were fixed too early, there would be a risk of jumps in the order of $10^{-7}$ in mass values of precision mass standards, with consequences for the adjustment and verification of both class $E_{1}$ and $E_{2}$ weights according to OIML R 111 [7].

At its meeting in 2010 the CCM therefore recommended that the following conditions be met before the kilogram is redefined in terms of fundamental constants [8]: 


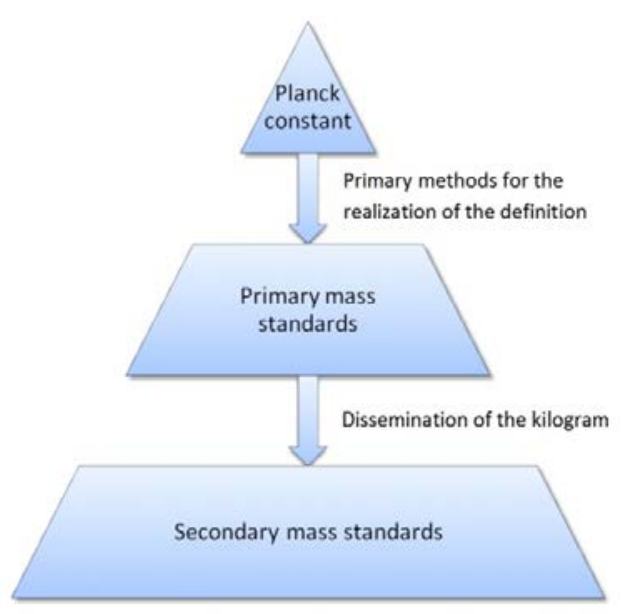

Figure 4. Proposed future realization of the kilogram with primary methods that would link primary mass standards to the fundamental constant $h$, followed by the classical way of dissemination using the primary mass standards to calibrate secondary ones.

1. At least three independent experiments, including work both from the watt balance and International Avogadro Coordination projects, yield values of the relevant constants with relative standard uncertainties not larger than $5 \times 10^{-8}$. At least one of these results should have a relative standard uncertainty not larger than $2 \times 10^{-8}$.

2. For each of the relevant constants, values provided by the different experiments should be consistent at the $95 \%$ level of confidence.

3. Traceability of BIPM prototypes to the international prototype of the kilogram should be confirmed.

In addition, CCM Recommendation G1 (2010) states the following:

4. The CODATA recommended values should be adopted for the relevant fundamental constants.

5. The associated CODATA relative standard uncertainties should be suitably considered when the initial uncertainty is assigned to the international prototype of the kilogram.

6. A pool of reference standards should be established at the BIPM to facilitate the dissemination of the redefined kilogram.

7. The BIPM and a sufficient number of National Metrology Institutes should continue to operate, develop or improve facilities or experiments that allow the realization of the kilogram to be maintained with a relative standard uncertainty not larger than $2 \times 10^{-8}$.

8. The uncertainty component arising from the practical realization of the unit should be suitably taken into account.

The above CCM recommendations, especially the first three, have not yet been met, but researchers are actively working to understand the differences in the experimental results and close the gaps. Currently, only two experiments have achieved published relative uncertainties smaller than $5 \times 10^{-8}\left(3.6 \times 10^{-8}\right.$ for the NIST watt balance [9] and $3 \times 10^{-8}$ for the International Avogadro Coordination experiments [10]). These results are
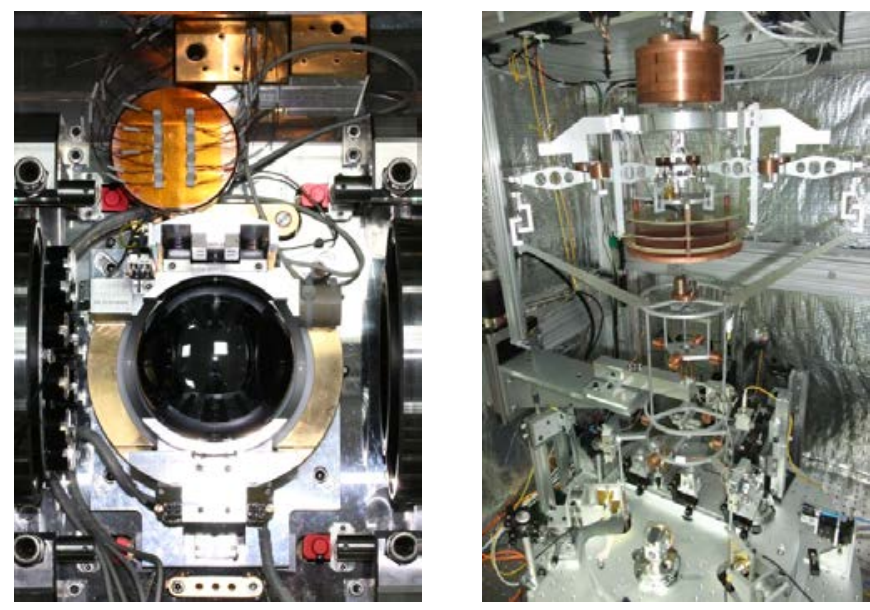

Figure 5. Existing primary methods for the realization of the new kilogram are the "Avogadro method" (or "X-ray Crystal Density (XRCD) method") and the "Watt balance method". Left: View of a single crystal silicon sphere. The diameter of the sphere (about $90 \mathrm{~mm}$ ), and hence, its volume is measured with a Fizeau interferometer, which forms part of the XRCD method (source: PTB Braunschweig, Germany). Right: View into the BIPM watt balance [6].

discrepant by $1.7 \times 10^{-7}$ as can be seen in Figure 6. The discrepancy between the recently published result of the NRC watt balance [11] and that of the NIST watt balance is even larger and amounts to about $2.6 \times 10^{-7}$, see Figure 6 .

\section{CONSEQUENCES FOR MASS METROLOGY}

While there is no doubt that a redefinition of the kilogram is desirable from the point of view of fundamental physics, the practical implications of a redefinition must be carefully considered beforehand in order to avoid negative consequences, not only for high-precision mass measurements, but also for the SI in general. Considering the extraordinary importance of weighing instruments, mass comparators and mass standards used in trade, industry and science, and also in daily life, the possible practical consequences and negative implications of a premature or even wrong decision could be tremendous. Despite its limitations, the current definition of the kilogram has quite successfully guaranteed up to now, that - all over the world - high-precision mass standards and weights of accuracy classes $E_{2}, E_{1}$ and even better are calibrated and used in the global market without any problems. Based on the CIPM Mutual Recognition Agreements (MRA) [15] calibration certificates have meanwhile achieved a high degree of worldwide acceptance.

A thorough examination of the realization, dissemination chain and uncertainty propagation for the redefined kilogram shows that $[16,17]$ :

- If all the above CCM recommendations are closely observed and met, no serious changes in the calibration chain of mass standards will occur, including high-precision mass standards and weights of class $\mathrm{E}_{1}$,

- However, even if the CCM recommendations are met, the uncertainty values in the "calibration and measurement capabilities" (CMCs) of NMIs will increase by up to a factor of 2, at least in the range from $100 \mathrm{~g}$ to $10 \mathrm{~kg}$, see Figure 7,

- If the CCM recommendations were not met, mass standards of high accuracy with a relative uncertainty smaller than or equal to $4 \times 10^{-8}$, as presently offered by NMIs, would no longer be available, and 


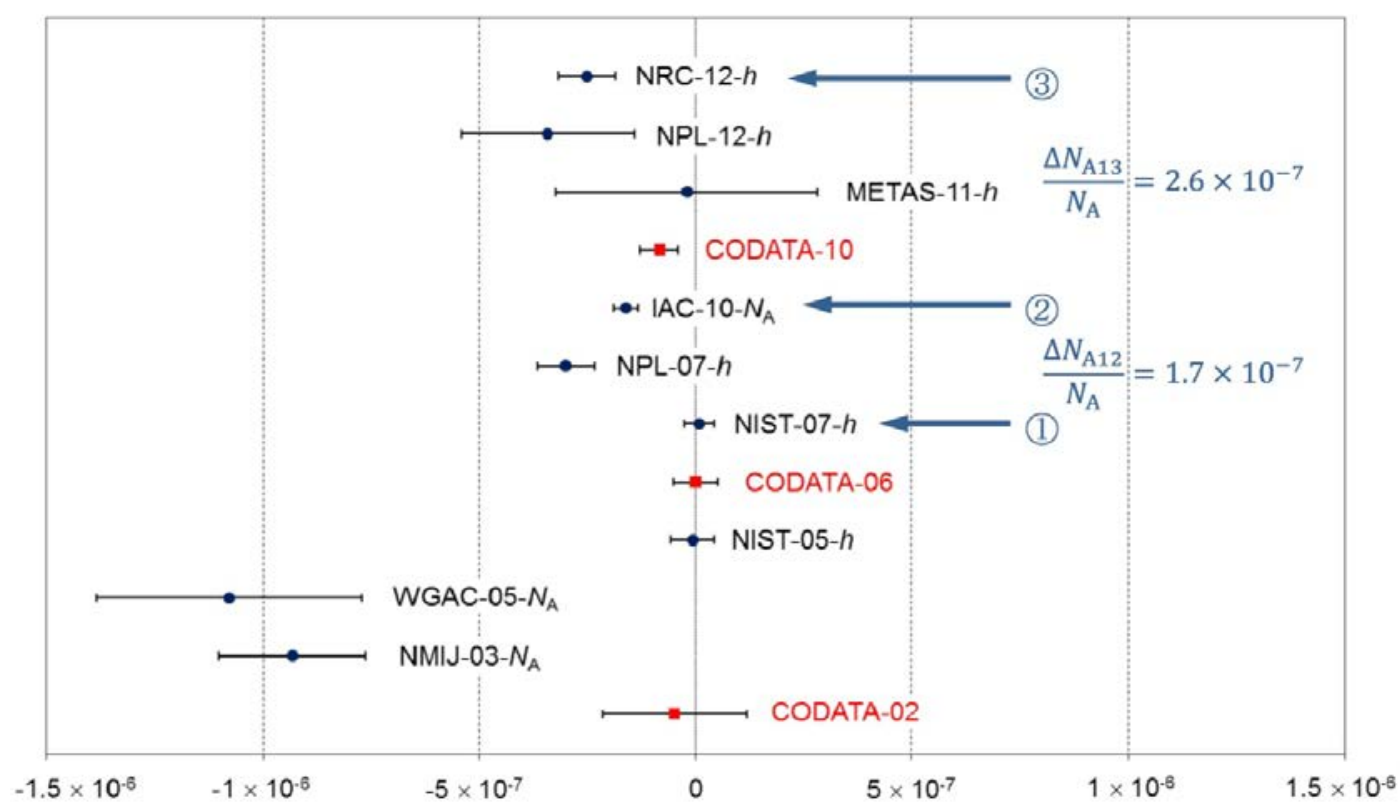

Figure 6. Measurement results for the Avogadro constant $N_{\mathrm{A}}$ with standard uncertainties $(k=1)$, represented as relative deviations from the CODATA 2006 value $\left(N_{\mathrm{A}}=6.02214179(30) \times 10^{23} \mathrm{~mol}^{-1}\right)$. The results for the Planck constant, $h$, (watt balance) and for $K_{\mathrm{J}}$ (voltage balance) have been converted by means of the CODATA 2006 constants. Explanations: "NPL-07- $h$ ", for example, means: NPL's result in 2007 for a measurement of $h$. WGAC: Working Group Avogadro Constant, IAC:

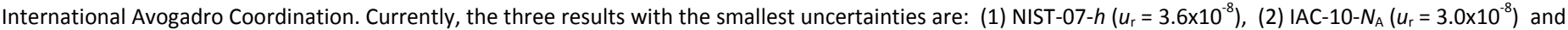
(3) NRC-12- $h\left(u_{r}=6.5 \times 10^{-8}\right)$. Measurement results and CODATA values according to the data published in $[5,12,13]$.

- There would be the risk that accredited calibration laboratories would no longer be able to calibrate class $\mathrm{E}_{1}$ weights according to OIML R 111; this would rather be the exclusive task of certain NMIs, which have sufficiently accurate mass standards available.

There is another risk of a premature redefinition if one or more of the above CCM conditions are not closely observed or are ignored. Most likely the CODATA recommended values will be the basis for fixing the relevant fundamental constants, which is the Planck constant $b$ in case of the kilogram. Figure 8 shows the long-term mass changes of platinum-iridium kilogram prototypes since 1889 [18]. This is contrasted with the values for $b$ resulting from the CODATA adjustments since 1998 [5, 19]. The CODATA values jump within four years by about $1 \times 10^{-7}$ which is a factor two worse than the assumed instability of the IPK during the past hundred years. It is obvious that jumps of such an order must at all be avoided for mass calibrations. The consequence for high-precision mass standards could be that the respective calibration certificates would have to be re-issued by the mass calibration laboratories responsible.

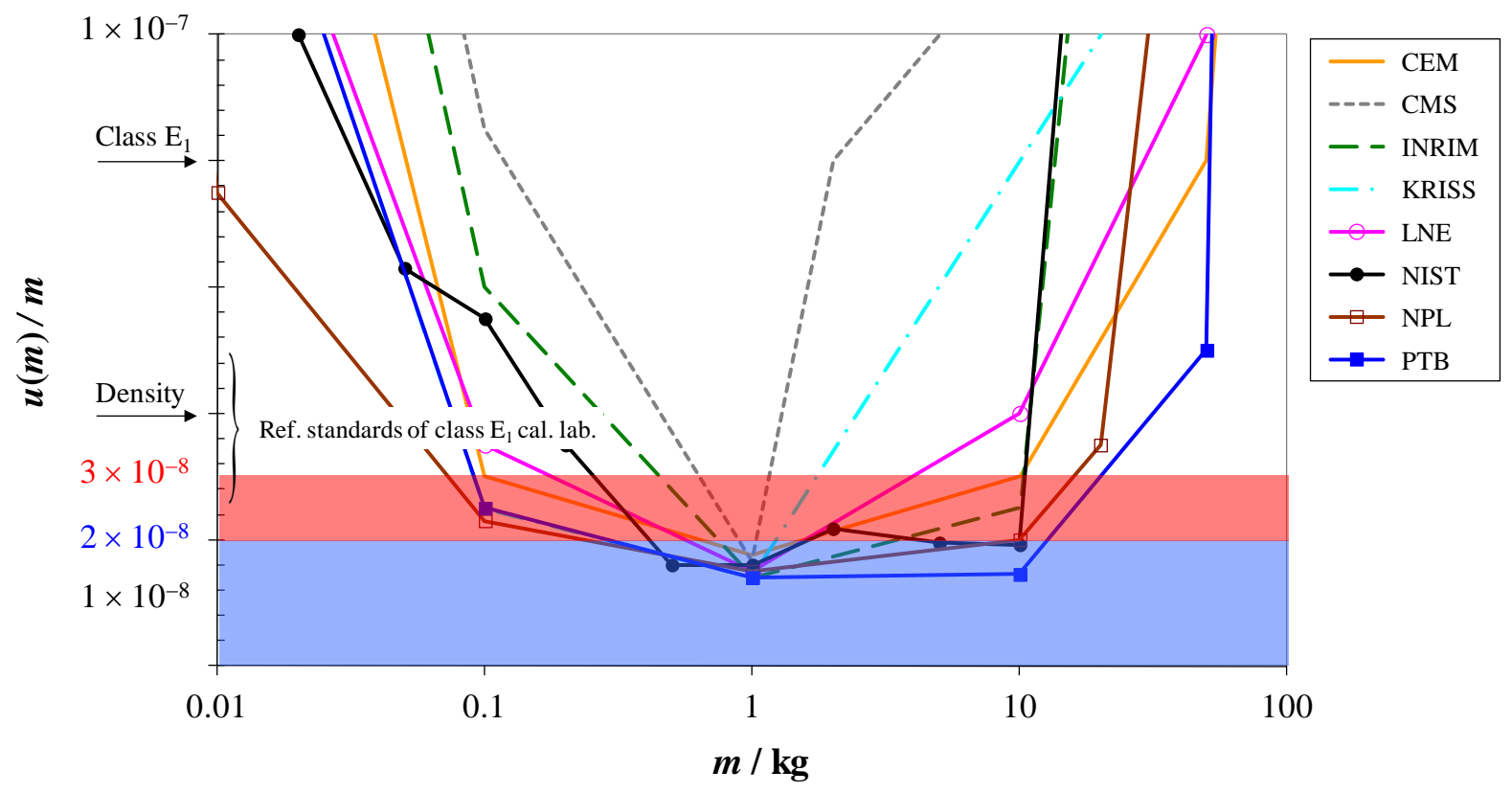

Figure 7. Calibration and Measurement Capabilities (CMCs) for mass standards in the range from $10 \mathrm{~g}$ to $100 \mathrm{~kg}$ of eight selected NMIs [14] compared with different uncertainty limits for the realization of a redefined kilogram and relative standard uncertainties of the best primary density standards (see pointer "Density"), for reference standards of class $E_{1}$ calibration laboratories (see range indicated), and for class $E_{1}$ weights (see pointer "Class $E_{1}$ "). 


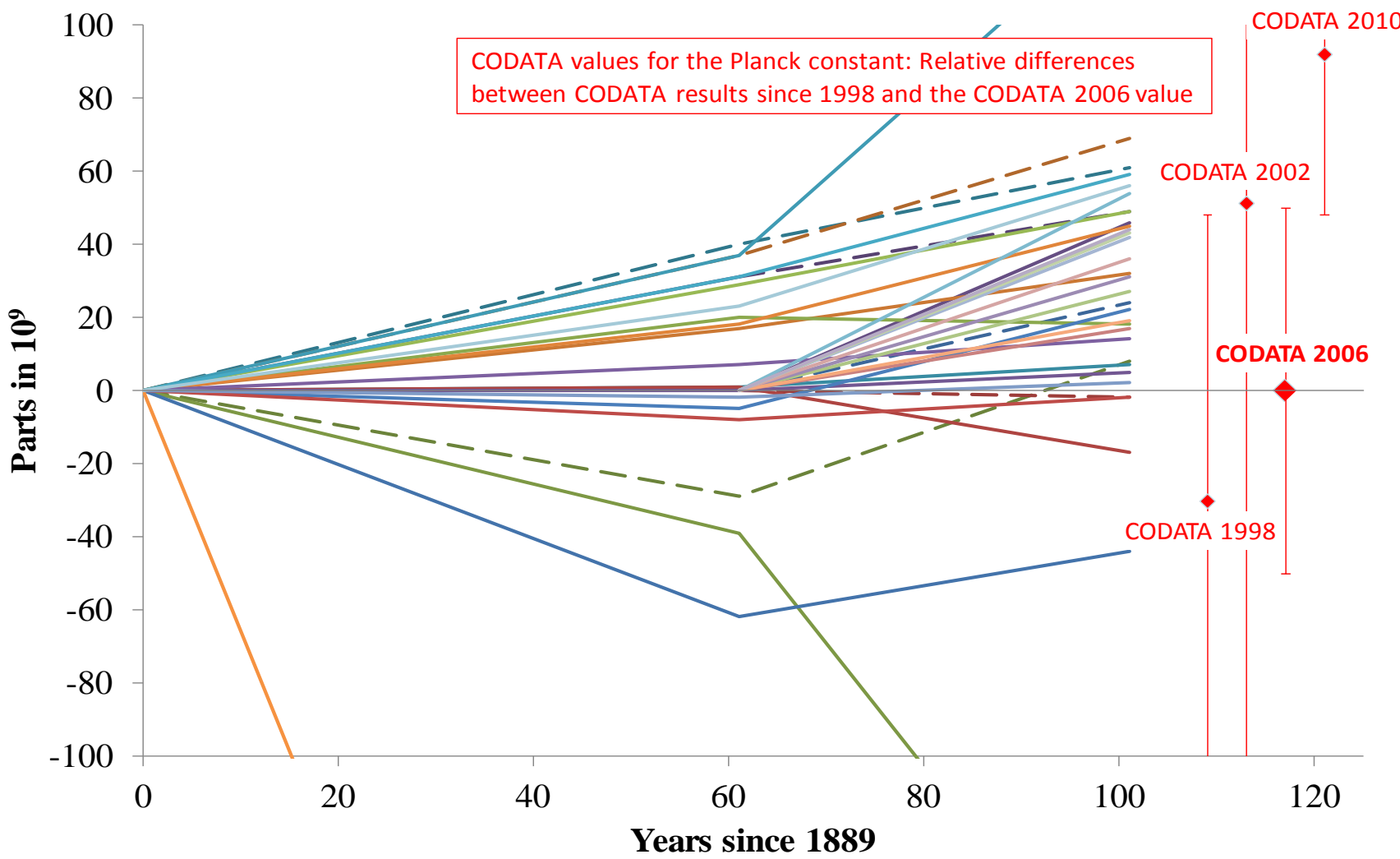

Figure 8. Mass changes of the six official copies (dashed lines) and national prototypes nos. 2 to 55 against the IPK since 1889 [18], compared with relative changes of CODATA values for the Planck constant, $h$, since $1998[5,12,13]$, where the CODATA 2006 value was (arbitrarily) chosen as a reference value.

In response to Resolution 1 of the CGPM, the International Organization for Legal Metrology (OIML) carried out an inquiry amongst its member states and its relevant Technical Committees in 2012, in order to explore in more detail the possible practical consequences of a revised SI in general, and a redefined kilogram in particular. The results of that inquiry and the official statement of the OIML according to CIML Resolution no. 25 (2012) [20] were recently published [19].

Also in response to Resolution 1 of the CGPM, the European Weighing industry association (CECIP), an important stakeholder in the field of mass measurement, submitted to the BIPM a position paper on the revision of the SI in 2012 [21]. The paper clearly addresses the potential negative impacts that a premature redefinition of the kilogram could have on precision mass measurements, but also on the SI in general. In addition, CECIP requests to improve the proposed new definitions, especially that of the kilogram, in order that the new SI remains understandable for its users.

\section{SUMMARY AND CONCLUSION}

This paper outlines the background and most important changes that are proposed to revise the present SI.

It also describes the current status of the redefinition of the kilogram, the relevant experiments, and the respective recommendations of the CCM that are intended to ensure the continuity of mass values before and after the redefinition of the kilogram at a level of about $2 \times 10^{-8}$.

It can be shown that if all CCM conditions and recommendations are closely observed and met, no serious changes in the calibration chain of mass standards would occur, although an increase of the best uncertainties of the CMC entries by up to a factor of two must be accepted.

If the conditions of the CCM are not closely observed, however, especially if there is not enough patience to wait for the results of the very active experimental work which is ongoing across the globe to further improve the data for $b$ and $N_{\mathrm{A}}$ as regards precision, uncertainty and consistency, there is a high risk that a premature redefinition of the kilogram could lead to significant jumps in the order of $10^{-7}$ in the mass values of high-precision mass standards having relative uncertainties as small as $4 \times 10^{-8}$ and less. As a consequence, accredited industrial calibration laboratories could even loose their ability to calibrate class $\mathrm{E}_{1}$ weights according to OIML R 111.

\section{REFERENCES}

[1] "On the possible future revision of the SI", http://www.bipm.org/en/si/new_si/.

[2] http://www.bipm.org/utils/en/pdf/24_CGPM_Resolution_1.pdf.

[3] http://www.bipm.org/en/si/new_si/what.html.

[4] Draft Chapter 2 for SI Brochure, following redefinitions of the base units", CCU, 29 September 2010, http://www.bipm.org/ /utils/common/pdf/si_brochure_draft_ch2.pdf .

[5] P. J. Mohr, B. N. Taylor and D. B. Newell, "CODATA recommended values of the fundamental physical constants: 2010”, Rev. Mod. Phys., Vol. 84, No. 4, pp. 1527- 1605, 2012.

[6] http://www.bipm.org/en/scientific/elec/watt_balance/wb_bip m.html.

[7] OIML R 111-1, "Weights of classes $\mathrm{E}_{1}, \mathrm{E}_{2}, \mathrm{~F}_{1}, \mathrm{~F}_{2}, \mathrm{M}_{1}, \mathrm{M}_{1-2}, \mathrm{M}_{2}$, $\mathrm{M}_{2-3}$ and $\mathrm{M}_{3}$, Part 1: Metrological and technical requirements", ed. 2004, http://www.oiml.org/publications/R/R111-1-e04.pdf.

[8] CCM Recommendation G 1 (2010), "Considerations on a new definition of the kilogram", http://www.bipm.org/utils $/$ common/pdf/CCM12.pdf\#page=23. 
[9] R. L. Steiner, E. R. Williams, R. Liu and D. B. Newell, "Uncertainty improvements of the NIST electronic kilogram", IEEE Trans. Instrum. Meas., vol. 56, pp. 592-596, 2007.

[10] B. Andreas, Y. Azuma, G. Bartl, P. Becker, H. Bettin, M. Borys, I. Busch, M. Gray, P. Fuchs, K. Fujii, H. Fujimoto, E. Kessler, M. Krumrey, U. Kuetgens, N. Kuramoto, G. Mana, P. Manson, E. Massa, S. Mizushima, A. Nicolaus, A. Picard, A. Pramann, O. Rienitz, D. Schiel, S. Valkiers, A. Waseda, "Determination of the Avogadro constant by counting the atoms in a ${ }^{28} \mathrm{Si}$ crystal", Phys. Rev. Lett., vol. 106, 030801, 2011.

[11] A. G. Steele, J. Meija, C. A. Sanchez, L. Yang, B. M. Wood, R. E. Sturgeon, Z. Mester and A. D. Inglis, "Reconciling Planck constant determinations via watt balance and enriched-silicon measurements at NRC Canada", Metrologia, vol. 49, pp. L8-L10, 2012.

[12] P. J. Mohr and B. N. Taylor, "CODATA recommended values of the fundamental physical constants: 2002", Rev. Mod. Phys., Vol. 77, No. 1, pp. 1-107, 2005.

[13] P. J. Mohr, B. N. Taylor and D. B. Newell, "CODATA recommended values of the fundamental physical constants: 2006", J. Phys. Chem. Ref. Data 37, pp. 1187-1284, 2008.
[14] Calibration and Measurement Capabilities - CMCs, http:// kcdb.bipm.or g/appendixC/.

[15] CIPM Mutual Recognition Arrangement (MRA): http://www.bipm.org/en/cipm-mra/.

[16] M. Gläser, M. Borys, D. Ratschko and R. Schwartz, "Redefinition of the kilogram and the impact on its future dissemination", Metrologia, vol. 47, pp. 419-428, 2010.

[17] R. Davis, "Proposed change to the definition of the kilogram: Consequences for legal metrology", OIML Bulletin, vol. LII, pp. 5-12, 2011.

[18] G. Girard, "The third periodic verification of national prototypes of the kilogram (1988-1992)", Metrologia, vol. 31, pp. 317-336, 1994.

[19] R. Schwartz, P. Richard, C. Ehrlich., Y. Miki, "The proposed new SI and consequences for legal metrology", Bulletin OIML, Vol. LIV, No.1, pp. 5-15, 2013.

[20] http://www.oiml.org/download/docs/ciml/47_ciml_resolutions english.pdf.

[21] CECIP, Position on possible future revision of the International System of Units - SI, http://www.bipm.org/ws/CCM/ MeP_2012/Allowed/November_2012/06A_CECIP_position_o n_SI_15_05_2012.pdf. 